significant proportion of cases this name differed from that deemed appropriate when all the properties of the organism were considered; the discrepancies arose either because an organism was atypical for its group in a characteristic used in the key - all microbiologists know well the atypical organism - or because of difficulty in interpreting test results. The authors recognise the problem, for they provide a useful summary of the features which distinguish each species from all, or all but a named few, other species; and this helps the inexperienced to detect errors.

In spite of this drawback, the Guide provides much useful information which is particularly valuable for the expert, for it brings together in one place much information published since the 1970 edition of The Yeasts. Since then many new species have been described and massive reorganisations of taxonomy have been recommended, accepted by the authors and incorporated into the keys. Although this makes it difficult to use the Guide in conjunction with the 1970 edition, as recommended by the authors, the ready accessibility of this information is nevertheless most welcome. One is tempted to wish that the authors had produced a still more substantial monograph which would have been of great value to the beginner and to the expert alike.

B.H. Kirsop is Head of the Microbiology Division at the ARC Food Research Institute, Norwich, UK.

\section{Dealing with information in the nervous system}

\section{H. B. Barlow}

Information Transmission in the Nervous System. By A. M. Uttley. Pp. 111. (Academic: London and New York, 1979.) $£ 7.50 ; \$ 15.50$.

IT is obvious that Pavlov's dogs, who salivated when a bell sounded, had in some way formed an assessment of the conditional probability of food, given the sound of a bell; because the dogs' past history had been controlled so that this probability was high, they salivated on the sound of a bell, without waiting for the food. In this sense, the nervous system must be able to compute conditional probabilities, and this is the starting point for the work, stretching back over 30 years, that Albert Uttley summarises in this book.

I think he is right in giving central importance to the question how conditional probabilities are assessed in the nervous system, and I also like another feature of his approach. Given its central importance, he says, let us explore the hypothesis that modifiable synapses in the nervous system are commonly arranged in such a way that the modified synapse stores a conditional probability. The book is a development of this theme, and centres on the "informon", an element for which a stored variable, $Y_{i}$, representing the effectiveness of a synapse, is given by

$\mathrm{Y}_{i}=-k \log \left(P\left(X_{i}\right.\right.$ and $\left.\left.Y\right) /\left(P\left(X_{i}\right) \times P(Y)\right)\right)$ where $k$ is a positive constant, and and $Y$ are two binary signals, the $X^{\prime}$ s usually representing inputs and $Y$ an output. $Y_{i}$ is thus the negative of Shannon's Mutual Information Function for $X_{i}$ and $Y$. Before going into further details two warning are required.

First, the title might mislead the unwary reader. It is not a general treatise on how information, in the general sense, is transmitted in the nervous system. Rather, the title was chosen, I suspect, to emphasise that "informons" adjust themselves in accordance with "information" in the specialised Shannon sense, rather than just messages; they store the conditional probabilities that enable them to deal effectively with information.

The second warning is that, although I am sympathetic to the author's general purpose and train of though, I did not find the book easy to read and follow. The author has perhaps lived with the ideas so long that he no longer has the perspective necessary in order to explain them effectively to newcomers.

The book contains eleven short chapters. The first four explain the concepts and illustrate how a theoretical "informon" might be realised by a nerve cell with modifiable and unmodifiable synaptic imputs. The next two chapters match the conceptual informon's behaviour against that of granule cells in the hippococampus, as shown by the work of Bliss and Lomo in rabbit and Douglas and Goddard in rat. He then deals with classical conditioning, the use of informons for pattern recognition, the interpretation of plasticity in the visual cortex, and the extension of the ideas to operant conditioning.

The book is a valiant and rather isolated effort in an original direction. It should be read by those who seek an explanation for quite complex psychological facts in elementary properties of synapses and nerve cells, and it thus forms a contrast with explanations for those same facts in terms of "programs" that could be realised by any kind of computing machinery. Conditional probabilities and Shannon's Information are quantities that form conceptual bridges between the two approaches, and the most valuable feature of Uttley's work may be to show how much can be achieved directly by neural hardware adapted to computing these quantities

H. B. Barlow is Professor in the Physiological Laboratory, University of Cambridge, UK.

\section{Objectives of agricultural policy}

R. F. Lord

Government and Agriculture: A Spatial Perspective. By I. Bowler. Pp.127. (Longman: Harlow, 1979.) Paperback $£ 4.50$.

DR BOWLER is one of a growing band of geographers concerned not only to describe, but also to hypothesise. In this book he takes the view that "a spatial perspective can provide additional insights into the government - agriculture relationship"'. That he only partially succeeds in his task is due largely to the fact that objectives of agricultural policy rarely contain a specifically spatial dimension and that even where they do, statistically acceptable data are seldom available on a spatial basis in order to measure achievement.

The first part of the book drawing on experience in a number of developed countries, with a wealth (surfeit?) of references, considers how agricultural policy has been influenced by the changing nature of the "agricultural problem" and concludes that for the most part there has been a failure to achieve the policy goals so optimistically set by governments.

The second part of the book relates solely to the development of UK agricultural policy in the post-war period up to 1973. Only in the last two chapters (6 and 7) is attention devoted to the spatial aspects of the problem (which considering the title of the book is surprising). Generally the ineffectiveness of measures in a spatial sense is demonstrated. However, $\mathrm{Dr}$ Bowler's concern for the spatial dimension is justified in his concluding paragraph where he refers to the need for more research of this type if problems of formulating adequate regional policies in the EEC are to be overcome.

R. F. Lord is Head of the Economics and Management Department in the School of Agriculture, University of Edinburgh, UK. 